\title{
Pointing large antennas using the conical scan technique
}

\author{
L. Olmi ${ }^{\star}$ and M.M. Davis \\ Cornell University, NAIC, Arecibo Observatory, P.O. Box 995, Arecibo, Puerto Rico 00613, U.S.A.
}

Received May 13; accepted September 11, 1997

\begin{abstract}
We describe an alternative way of pointing (large) radiotelescopes using a method derived from radar tracking techniques, and generally known as conical scanning. It can be accomplished by using either a feed that is mechanically (or electrically) displaced from the boresight axis, or by fixing the feed and translating or rotating a tilted subreflector. The optimum set-up for radioastronomical application depends upon the antenna configuration, the feed mount, and the sizes/weights of the moving parts involved. In the case of the new Gregorian configuration for the Arecibo telescope, the mechanical and electromagnetic configuration suggests the use of combined motions of the tertiary reflector in elevation and the feed turret in cross-elevation. We analyse several methods of implementing the conical scan technique and evaluate them through numerical simulations.
\end{abstract}

Key words: techniques: pointing - telescopes methods: analytical

\section{Introduction}

The National Astronomy and Ionospheric Center (NAIC $\left.{ }^{1}\right)$ is currently completing the second Upgrade of the 305-m Arecibo Observatory (AO) radiotelescope. This includes the construction of a 26-m Gregorian enclosure on the feed arm 137-m above the reflector. 19.8-m secondary and 7.6-m tertiary subreflectors have been installed inside this aerodynamic enclosure. This system replaces one of the previous two carriage houses, and provides correction for spherical aberration. One of the main achievements of the Gregorian upgrade will thus be to extend the frequency

Send offprint requests to: M. Davis

* Present address: LMT Project and FCRAO, 815 Lederle G.R.C., University of Massachusetts, Amherst, MA 01003 , U.S.A.olmi@fcrao1.phast.umass.edu

1 The National Astronomy and Ionospheric Center is operated by Cornell University under a cooperative agreement with the National Science Foundation. coverage of the Arecibo telescope to the highest practicable frequencies limited by the accuracy of the primary reflector $(\simeq 10 \mathrm{GHz})$, with considerable advantages for both molecular spectroscopy and pulsar astronomy (see Kildal et al. 1994 and Olmi \& Baan 1997).

The goal of the upgrade is to have a 305-m telescope which performs well at radio wavelengths as short as $3 \mathrm{~cm}$. As the main beam width of large diameter antennas becomes very narrow with increasing operating frequency, the highest pointing accuracy is required. In the case of Arecibo, positioning the $\simeq 32^{\prime \prime}$ beam at $10 \mathrm{GHz}$ will require a pointing accuracy of $5^{\prime \prime}$ or better.

The conical scan method described below can be used as a fast, precise alternative to the widely-used cross-scan or five-point map techniques to measure pointing errors. Conical scanning is also known as sequential-lobe comparison, and is a technique widely used in tracking-radar systems (Skolnik 1970). It locates the center of the image through, e.g., mechanical nutation of a single feed that is displaced from the axis. The phase center of the feed is moved along the circumference of a circle which is centered on the optical axis (for a definition of the latter see below). A constant return occurs for a target on the boresight, and a modulated signal return occurs for a target that is off axis. This modulation, when properly compared in phase with the reference signal generated by the nutating mechanism, leads to the vectorial pointing error: the modulation amplitude gives the size of the correction, and its phase angle gives the direction.

Continuous beam scanning can be accomplished by mechanically moving the feed of an antenna, as the antenna beam will move off axis when the feed is moved off the focal point. The feed is typically moved in a circular path around the focal point, causing a corresponding movement of the antenna beam in a circular path around the target. Conical scanning can also be accomplished by fixing the feed and moving a subreflector. At Arecibo we plan to combine movement of the feed turret in azimuth and the tertiary subreflector in zenith angle.

A very significant advantage arises from the very low mass of the feed turret and tertiary compared to the 200ton feed arm and 75-ton Gregorian enclosure. The latter 
are used to track the nominal source location, while the turret and tertiary provide small, rapid motions, not feasible with their more ponderous counterparts. In addition, movement of the feed turret by a fixed amount provides a fixed great circle motion of the beam on the sky, in strong contrast to motion of the feed-arm, which must increase its offsets proportional to $\csc z$, where $z$ is the zenith angle, for a fixed great-circle beam motion. Hence, one of the principal advantages of this method is the absence of tracking problems near the zenith.

An alternative technique to sequential-lobe comparison in radar tracking applications is simultaneous-lobe comparison (or monopulse) which does not require sequential movement of the antenna beam in a path around the "target", and utilizes two (or more) different beams at the same time. The beams can be obtained either by using an array of physically distinct feeds or generating them electromagnetically, by using a multimode monopulse feed (Skolnik 1970; Sherman 1984). The obvious advantage of the monopulse technique is the ability to obtain complete angle-error information on a single pulse, or integration cycle in the case of astronomical applications, whereas conical scanning operates through a time-dependent scan.

However, in addition to other problems which include linearity in the response and cross-talk in the different output channels, the monopulse technique has some fundamental drawbacks in its radioastronomical use which are: (i) the need of a completely separate feed specifically designed for this purpose; (ii) its use at just one frequency band, and (iii) the need to accomodate this special feed where space is at a premium, as on the rotating platform which hosts the feeds in the upgraded Arecibo telescope. Instead, the use of the actual observing feeds in the sequential-lobe technique guarantees that any offsets peculiar to these feeds are included in the pointing correction. Therefore, in the present work we shall discuss the conical scan technique only.

The conical scan technique has been used for years at the Deep Space Network (DSN) antennas (Abichandani \& Ohlson 1981; Ohlson \& Abichandani 1982; Abichandani 1983 and Eldred 1994) and at the Parkes radiotelescope (Wark \& Wright 1990) for the main purpose of tracking spacecraft. The references cited above give both a general and detailed description of the conical scan method, with respect to the tracking problem, including optimization techniques (Eldred 1994). In this work we want to enhance those aspects of the conical scan technique which are more important to observe normal radio sources, by also using quantities which are familiar to radio astronomers. Furthermore, we compare alternative retrieving methods, including some analytic ones, and also discuss the implications of two important aspects of "real" antennas: the presence of sidelobes and the ellipticity of the antenna beam.

In Sect. 2 we thus list our main assumptions and derive the antenna temperature for a conical scan. Then, in
Sect. 3, several retrieving algorithms for the pointing offsets are described, and we discuss their general properties. The results obtained with simulations carried out with these different methods are described in Sect. 4, where both Gaussian and non-Gaussian antenna-beams are considered. Our conclusions are listed in Sect. 5 and we include two appendices: Appendix A discusses the effects of a non-circular antenna beam, and a definition of the principal symbols can be found in Appendix B.

\section{General assumptions}

We will now give a brief description of the conical scan technique and of our plans to implement this for the upgraded Arecibo telescope. Let us start with the following simplifying assumptions (but see Sect. 4.3):

(i) the antenna is commanded to point its beam axis at a certain location, referred as the "target" throughout the remainder of this article. Due to many inherent pointing errors the antenna boresight does not point to the desired location on the sky. Instead, the instrumentally tracked point is the center of the scanning circle defined below;

(ii) we define the optical axis (sometimes called also the RF axis or beam axis) as the best-fit axis of the entire deformed reflector, as distinct from the geometrical axis of the undeformed theoretical reflecting system;

(iii) the beam is Gaussian and depends only on the angular distance ( $r$, see below) from the axis. Beams with elliptical cross-sections are considered in Appendix A;

(iv) the beam remains unchanged with elevation;

(v) we neglect for the moment the problem of the inner sidelobes, which will be discussed in Sect. 4.2;

(vi) we shall not consider the problem of beam-squint between opposite circularly polarized beams, i.e. in the case of polarized signals this is equivalent to considering a single polarization direction.

Given a point source located at angular great-circle offset $r$ from the beam center, a suitable representation of the value of the normalised antenna power pattern at position $r$ can be written as:

$P_{\mathrm{n}}(r)=\frac{W_{\mathrm{on}}(r)-W_{\text {off }}}{W_{\mathrm{src}}-W_{\text {off }}}=\exp \left(-k r^{2}\right)$

where $W_{\text {on }}(r)$ represents the measured receiver output power when the source is at position $r, W_{\mathrm{src}} \equiv W_{\mathrm{on}}(r=0)$ is the maximum recorded output power when the source is at the center of the beam, and $W_{\text {off represents the mea- }}$ sured power when no source is within the beam. For $r$ 
expressed in units of the full beamwidth at half maximum, FWHM (see Fig. 1), $k=4 \ln 2$; all other angular distances will hereafter be expressed in these units, unless explicitly stated otherwise. Equation (1) implies that at some point during the procedures described in the next sections a measurement is taken of the power OFF-source.

During the conical scan the antenna beam moves in a circular path of radius $\beta$ centered on the optical axis. In general, $\beta$ is frequency-dependent. This fact can actually be used as a further aid in the practical implementation of the conical scan technique, but we shall not discuss it in this work, and we shall thus drop the frequency dependence and treat $\beta$ as a constant. Choosing a reference frame whose origin is the center of the scanning circle, the position of the beam center, $\left(X_{\mathrm{c}}, Y_{\mathrm{c}}\right)$, can be written as:

$\left\{\begin{array}{l}X_{\mathrm{c}}=\beta \cos \theta(t) \\ Y_{\mathrm{c}}=\beta \sin \theta(t)\end{array}\right.$

where we define $Y_{\mathrm{c}}$ and $X_{\mathrm{c}}$ to be the positions in elevation and cross-elevation, respectively, $\theta(t)$ is the conical scan angle (see Fig. 1), and $t$ is the time.

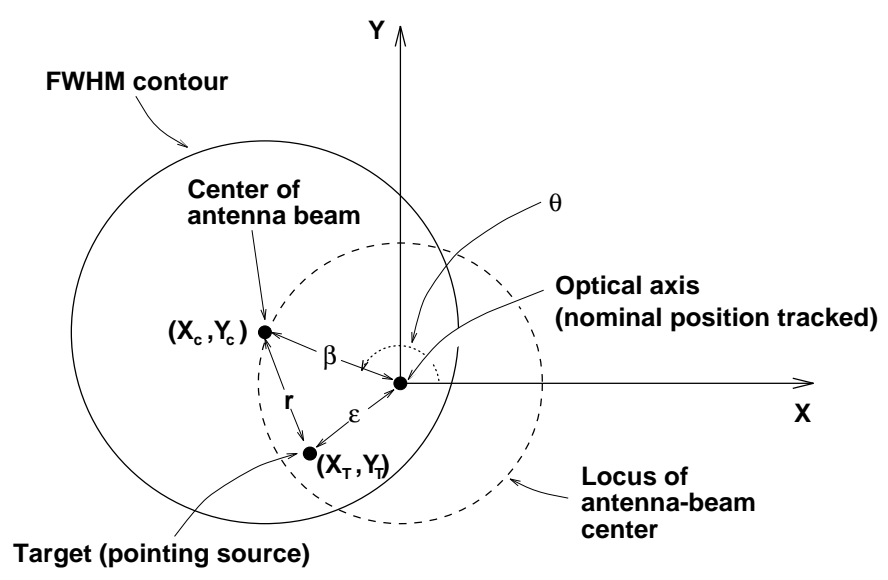

Fig. 1. Plane of the sky where the antenna beam FWHM contour (solid circle) and the path of rotation of the beam center (dashed circle), are shown. $\beta$ and $\epsilon$ are defined in the text

We assume that a pointing source, or target, having a negligible angular size with respect to the $32^{\prime \prime}$ beam at $10 \mathrm{GHz}$, is located at position $\left(X_{\mathrm{T}}, Y_{\mathrm{T}}\right)$ with respect to the center of the scanning circle, $(0,0)$. Then from Fig. 1 it is easily shown that

$r^{2}=\beta^{2}+\epsilon^{2}-2 \beta\left[X_{\mathrm{T}} \cos \theta(t)+Y_{\mathrm{T}} \sin \theta(t)\right]$

where $\epsilon=\sqrt{X_{\mathrm{T}}^{2}+Y_{\mathrm{T}}^{2}}$ and $\beta=\sqrt{X_{\mathrm{c}}^{2}+Y_{\mathrm{c}}^{2}}$ are the angular distances, from the pointing direction (or, equivalently, the optical axis), of the target and the center of the beam, respectively.
The antenna temperature, $T_{\mathrm{A}}(r)$, when the source is at an angular distance $r$ from the optical axis can be measured in the usual way:

$T_{\mathrm{A}}(r)=\frac{W_{\mathrm{on}}(r)-W_{\mathrm{off}}}{W_{\text {off }}} T_{\text {sys }}$

where $T_{\text {sys }}$ is the on-source system temperature and $W$ is the measured power. Also, by definition

$T_{\mathrm{A}}^{\mathrm{src}} \equiv T_{\mathrm{A}}(r=0)=\frac{W_{\mathrm{src}}-W_{\mathrm{off}}}{W_{\text {off }}} T_{\mathrm{sys}}$

is the expected antenna temperature of the pointing source. From Eqs. (1), (4) and (5) we then obtain the known result that $T_{\mathrm{A}}(r) / T_{\mathrm{A}}^{\mathrm{src}}=P_{\mathrm{n}}(r)$ or, by Eq. (3):

$$
\begin{aligned}
T_{\mathrm{A}}(\theta ; \beta, \epsilon)= & T_{\mathrm{A}}^{\mathrm{src}} \exp \left[-k\left(\beta^{2}+\epsilon^{2}\right)\right] \times \\
& \exp \left[2 k \beta\left(X_{\mathrm{T}} \cos \theta+Y_{\mathrm{T}} \sin \theta\right)\right]
\end{aligned}
$$

where $\theta$ is implicitly assumed to be $\theta=\theta(t)$. Examples of Eq. (6) are shown in Fig. 3, and are discussed in Sect. 4.1.1.

\section{Retrieving algorithms}

The basic problem of the conical scan technique is how to retrieve the pointing errors, i.e. the best estimates of the two numbers $X_{\mathrm{T}}$ and $Y_{\mathrm{T}}$. One possibility is given by the simple fact that as conical scanning operates by displacing the antenna beam to successive positions, it is possible to measure the difference in the relative signal strength, expressed by $\Delta P_{\mathrm{n}}=P_{\mathrm{n}}(\theta+\Delta \theta ; \beta, \epsilon)-P_{\mathrm{n}}(\theta ; \beta, \epsilon)$. The signal difference, $\Delta P_{\mathrm{n}}$, is a function of the angular difference, $\Delta \theta$, and as we shall see below it can be used to calculate the pointing errors.

However, we shall first review the standard method, consisting of recording the antenna signal and computing sums weighted by sine and cosine factors. We then discuss two analytical methods which exploit the measurement of the signal difference. In each case, we assume that a total of $N$ measurements are taken in one $360^{\circ}$ scan, at $\Delta \theta$ intervals, each one lasting $\Delta t$ seconds.

We also note that the standard method, as discussed below, assumes that $T_{\mathrm{A}}^{\mathrm{src}}$ is known at the time of the pointing measurement. This may not always be the case, especially if the pointing source is variable and has not been observed for a long time. The two successive analytical methods, however, do not need the knowledge of $T_{\mathrm{A}}^{\mathrm{src}}$. This can be an important advantage when the flux density of the pointing source is poorly known a priori. However, we shall show that an inaccurate $T_{\mathrm{A}}^{\mathrm{src}}$ at most leads to overor underestimates in the amplitude of the pointing error, $\epsilon$, but still accurately estimates the ratio $Y_{\mathrm{T}} / X_{\mathrm{T}}$, i.e. the direction towards the source. $T_{\mathrm{A}}^{\mathrm{src}}$ is unimportant for small $\epsilon$, and hence a sequence of scans converges rapidly. 


\subsection{Standard method}

The standard method consists of directly fitting the antenna signal using Eq. (6). The target position $\left(X_{\mathrm{T}}, Y_{\mathrm{T}}\right)$ provides the variables and the best-fit model is found minimising the value of $\chi^{2}$. When this method is used without further simplifying Eq. (6), it is much slower than fitting the signal difference or using the dot-product technique, which we discuss below. However, the antenna signal is much less noisy than the signal difference and, being a numerical method, we can use any beam shape to fit the data, and not just a Gaussian beam as it is assumed in Eq. (6).

The standard fitting method is very simple in those cases where Eq. (6) can be linearized, and a much quicker least-square fit technique can be used, making this method more useful in all practical cases.

As $\beta$ and $\epsilon$ are constants, only the right-hand side exponential in Eq. (6) is time variable, for $\theta=\theta(t)$. Expanding this factor to first order:

$$
\begin{aligned}
\frac{T_{\mathrm{A}}(\theta ; \beta, \epsilon)}{T_{\mathrm{A}}^{\mathrm{src}}} \simeq & \exp \left[-k\left(\beta^{2}+\epsilon^{2}\right)\right] \times \\
& {\left[1+2 k \beta\left(X_{\mathrm{T}} \cos \theta+Y_{\mathrm{T}} \sin \theta\right)\right] }
\end{aligned}
$$

or, using an angle $\theta_{\epsilon}$ to define the unknown direction of the pointing offset $\epsilon$, such that

$$
\left\{\begin{array}{l}
X_{\mathrm{T}}=\epsilon \cos \theta_{\epsilon} \\
Y_{\mathrm{T}}=\epsilon \sin \theta_{\epsilon}
\end{array}\right.
$$

then, Eq. (7) can also be written as:

$$
\frac{T_{\mathrm{A}}(\theta ; \beta, \epsilon)}{T_{\mathrm{A}}^{\mathrm{src}}} \simeq \exp \left[-k\left(\beta^{2}+\epsilon^{2}\right)\right]\left[1+2 k \beta \epsilon \cos \left(\theta-\theta_{\epsilon}\right)\right] .
$$

We then see that the expansion to first order made in Eq. (7) is valid when $\epsilon \ll 1 / 2 k \beta$, i.e. $\epsilon \ll 0.9$ and $\epsilon \ll 0.3$ for $\beta=0.2$ and $\beta=0.6$, respectively (for the case $\beta=0$ see below). Solution of Eq. (7) for $X_{\mathrm{T}}$ and $Y_{\mathrm{T}}$ is straightforward; in fact, multiplication by $\cos \theta$ and $\sin \theta$, and then integrating with respect to $\theta$ between 0 and $2 \pi$ exactly cancels the constant and the orthogonal $(\cos \theta \sin \theta)$ terms, leaving:

$$
\left\{\begin{array}{l}
X_{\mathrm{T}}=\frac{\exp \left[k\left(\beta^{2}+\epsilon^{2}\right)\right] I_{1}^{\cos }}{2 k \beta T_{\mathrm{A}}^{\mathrm{src}}} \\
Y_{\mathrm{T}}=\frac{\exp \left[k\left(\beta^{2}+\epsilon^{2}\right)\right] I_{1}^{\mathrm{sin}}}{2 k \beta T_{\mathrm{A}}^{\operatorname{sic}}}
\end{array}\right.
$$

where we have defined:

$$
\left\{\begin{array}{l}
I_{1}^{\cos }(\beta, \epsilon)=\frac{1}{\pi} \int_{0}^{2 \pi} T_{\mathrm{A}}(\theta ; \beta, \epsilon) \cos \theta \mathrm{d} \theta \\
I_{1}^{\sin }(\beta, \epsilon)=\frac{1}{\pi} \int_{0}^{2 \pi} T_{\mathrm{A}}(\theta ; \beta, \epsilon) \sin \theta \mathrm{d} \theta
\end{array}\right.
$$

$I_{1}^{\text {sin }}$ and $I_{1}^{\text {cos }}$ are recognizable as the first sine and cosine term of the Fourier series of $T_{\mathrm{A}}(\theta ; \beta, \epsilon)$. We give further details in Appendix A, where we also discuss the case of an elliptical antenna beam, and the useful information that can be obtained utilizing the Fourier series.

Equations (10) are the analytic least square fits to $X_{\mathrm{T}}$ and $Y_{\mathrm{T}}$ in the linearized Eq. (7), and make the standard fitting technique an analytical method similar to those discussed in Sect. 3.2.

We note that Eqs. (10) contain $\epsilon^{2}$ on their right-hand side, which is unknown. In the assumption of small pointing errors this is not a concern. However, the angular direction of the pointing offset, $\theta_{\epsilon}$, can be obtained with no approximations by taking the ratio of Eqs. (10) and using Eqs. (8):

$\tan \theta_{\epsilon}=\frac{I_{1}^{\sin }}{I_{1}^{\cos }}$

Also, this result is completely general. If the ratio of Eqs. (11) is taken with $T_{\mathrm{A}}(\theta ; \beta, \epsilon)$ being given by Eq. (6), rather than Eq. (7), it can be easily shown that $I_{1}^{\sin } / I_{1}^{\cos }=$ $Y_{\mathrm{T}} / X_{\mathrm{T}}=\tan \theta_{\epsilon}$.

Once the direction of the pointing offset is known, we can then solve Eqs. (10) for $\epsilon$ using its definition, $\epsilon=$ $\sqrt{X_{\mathrm{T}}^{2}+Y_{\mathrm{T}}^{2}}$ :

$\epsilon^{2} \exp \left(-2 k \epsilon^{2}\right)=\frac{\exp \left(2 k \beta^{2}\right)}{(2 k \beta)^{2}} \frac{\left[\left(I_{1}^{\cos }\right)^{2}+\left(I_{1}^{\sin }\right)^{2}\right]}{\left(T_{\mathrm{A}}^{\mathrm{src}}\right)^{2}}$

which can be numerically solved for $\epsilon$ if one can discriminate between the double-valued solution.

One method is to use an initial ON-OFF measurement. This is a sensitive estimate if $\epsilon$ is large, compared to the values leading to the approximation contained in Eq. (7), which cannot be used in this case. From Eq. (6) for $\beta=0$, the ratio of measured to expected antenna temperature is:

$\frac{T_{\mathrm{A}}(\beta=0, \epsilon)}{T_{\mathrm{A}}^{\mathrm{src}}} \equiv R_{\mathrm{T}}=\exp \left(-k \epsilon^{2}\right)$

and it is thus straightforward to obtain:

$\epsilon=\sqrt{\frac{1}{k} \ln \frac{1}{R_{\mathrm{T}}}}$

For $R_{\mathrm{T}}<0.8$ the pointing error is larger than $1 / 4$ beamwidth. In such cases the approximation used in Eq. (7), which leads to Eq. (13), is not as good as that from Eq. (15). However, the value of $\theta_{\epsilon}$ calculated by Eq. (12) is totally independent of the approximation used in Eq. (7), as stated above, provided that the beam is Gaussian.

The telescope pointing direction should be changed using $\theta_{\epsilon}$ and the estimate of $\epsilon$ from Eq. (15), until the pointing error is less than $1 / 4$ beamwidth. Once $R_{\mathrm{T}}>0.8$ Eq. (13) can be used. This estimate is more sensitive for small $\epsilon$ than Eq. (15), and is not subject to baseline errors, as it uses only the variation of $T_{\mathrm{A}}$ during the conical 


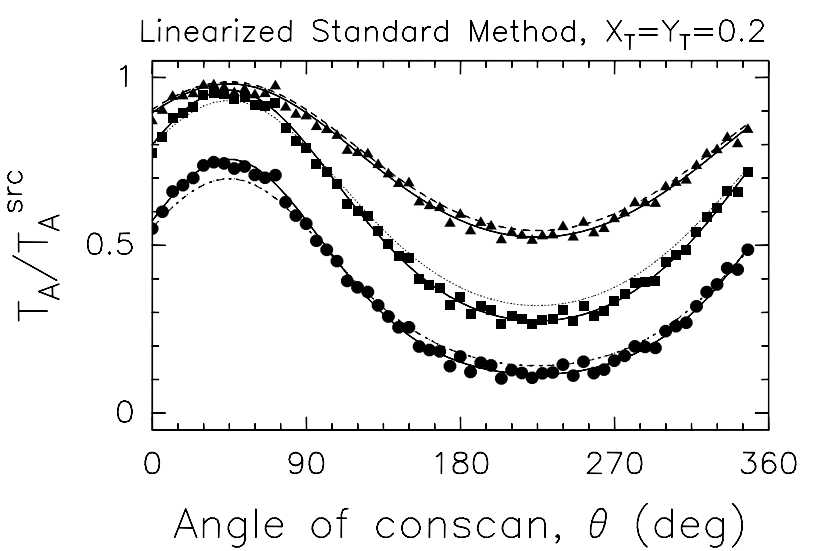

Fig. 2. Comparison between the fit to the antenna signal (points) utilising the linearized standard method (different types of dashed lines) and the $\chi^{2}$ fit (solid lines). The pointing error is fixed $\left(X_{\mathrm{T}}=Y_{\mathrm{T}}=0.2\right.$, or $\epsilon=0.28$, in FWHM units) and we consider three different values of $\beta$, the radius of the scan, in FWHM units: 0.2 (filled triangles and short-dashed line), 0.4 (filled squares and dotted line), and 0.6 (filled circles and long-dashed line). The data shown are for $\Delta t=0.1 \mathrm{~s}$ $\Delta \theta=6^{\circ}$ and a $\mathrm{SNR} \simeq 37$ (we have also assumed that the detection bandiwdth is $\Delta \nu=2 \mathrm{MHz}$ and the system temperature $T_{\text {sys }}=25 \mathrm{~K}$ )

scan, rather than its mean value. Hence, applying a sequence of pointing corrections will move the telescope in such a direction as to reduce the offset. If $R_{\mathrm{T}}<1 / 16$ the error is larger than 1 beamwidth. Such a result should be flagged to the telescope operator, as it may be the result of radio frequency interference (RFI) or an incorrect source position.

In Fig. 2 we show the difference between the leastsquares solutions and the approximation given by the use of Eq. (7), for various values of $\beta$ and for $X_{\mathrm{T}}=Y_{\mathrm{T}}=0.2$. We note that the maximum peak-to-peak amplitude in the response is obtained when $\beta=0.4$. In fact, the exact value for the conical scan radius corresponding to the maximum peak-to-peak amplitude is expected to be from Eq. (9) equal to $\beta=\sqrt{1 /(2 k)}=0.42$. Given the relatively low value of $\epsilon=0.28$ in Fig. 2 (see also the top panel of Fig. 5), the fits representing the results of the linearized standard method (dashed lines) lie reasonably close to those obtained by minimising a $\chi^{2}$ (solid lines), but in each case the linearized method underestimates the true error.

One potential pitfall of the conical scan method arises from the absence of any modulation for perfect pointing, which could be confused with total absence of the pointing source or some other breakdown in the system. However, an intentional offset of the boresight position by a small fraction of a beamwidth can provide a known modulation amplitude to prove that the system is functional.

\subsection{Analytical methods}

\subsubsection{Fitting the signal difference}

When $\Delta \theta$ is taken small enough $\left(\Delta \theta \lesssim 5^{\circ}\right)$, then $\Delta P_{\mathrm{n}}$ can be approximated using Taylor's expansion to first order and Eq. (6):

$$
\begin{aligned}
\Delta P_{\mathrm{n}} \simeq & \Delta \theta \frac{\mathrm{d} P_{\mathrm{n}}(\theta ; \beta, \epsilon)}{\mathrm{d} \theta}=2 k \beta P_{\mathrm{n}}(\theta, \beta) \times \\
& {\left[Y_{\mathrm{T}} \cos \theta-X_{\mathrm{T}} \sin \theta\right] \Delta \theta . }
\end{aligned}
$$

If we now define a normalised signal difference $\Delta S_{\mathrm{n}}=$ $\Delta P_{\mathrm{n}} /\left[2 k \beta P_{\mathrm{n}}(\theta ; \beta, \epsilon) \Delta \theta\right]$, one finally gets:

$\Delta S_{n}=\left[Y_{\mathrm{T}} \cos \theta-X_{\mathrm{T}} \sin \theta\right]$.

$\Delta S_{\mathrm{n}}$ can be a very noisy signal, because of the operations involved: the difference between two signals close in intensity, and the division by the signal itself, $P_{\mathrm{n}}(\theta)$ which, depending on the relative position of the source with respect to the center of the beam, can be very close to the noise level in some parts of the scanning circle.

Equation (17) can be compared with the measured signal difference, and because of its simple mathematical form it can be fit with a quick generalised linear least square technique, without having to numerically minimise a $\chi^{2}$-value, as we explained in Sect. 3.1. The results are discussed later in Sect. 4.1.2.

An important consequence of normalising the signal difference is that Eq. (17) is independent of the absolute flux density of the pointing source. Therefore, as we mentioned earlier in this section, the fitting method and the successive dot-product method are not affected by the (poorly known) values of $T_{\mathrm{A}}^{\mathrm{src}}$. Equation (17) is also independent of $\beta$, although the resulting signal-to-noise ratio (SNR) will depend on the radius of the conical scan, as is shown in Fig. 4.

\subsubsection{Dot-product method}

This method still assumes a Gaussian beam shape, although this is generally not the case. The angle-error estimate can be accomplished by the hardware, using a pair of phase-sensitive detectors with a reference input from the scan system. The phase detectors perform essentially as dot-product (DP, hereafter) devices with sine--wave reference signals at the frequency of scan and of proper phase to obtain the two numbers $X_{\mathrm{T}}$ and $Y_{\mathrm{T}}$. This can be easily seen analytically by multiplying the normalized signal difference by a sine-wave, $\sin (\theta+\phi)$, obtaining:

$$
\begin{aligned}
\Delta S_{\mathrm{n}} \sin (\theta+\phi)= & \frac{1}{2}\left[Y_{\mathrm{T}} \sin (2 \theta+\phi)+X_{\mathrm{T}} \cos (2 \theta+\phi)+\right. \\
& \left.Y_{\mathrm{T}} \sin (\phi)-X_{\mathrm{T}} \cos (\phi)\right]
\end{aligned}
$$

where $\phi$ is an instrumental phase factor. By filtering out the two oscillating components in the previous expression (see Sect. 4.1.3) one is left with:

$$
\Delta S_{n} \sin (\theta+\phi)=\frac{c}{2}\left(Y_{\mathrm{T}} \sin \phi-X_{\mathrm{T}} \cos \phi\right)
$$


which gives $Y_{\mathrm{T}}$ and $X_{\mathrm{T}}$ for $\phi=\pi / 2$ and $\phi=\pi$, respectively; $c$ is a numerical factor depending on the filtering process (see Sect. 4.1.3).

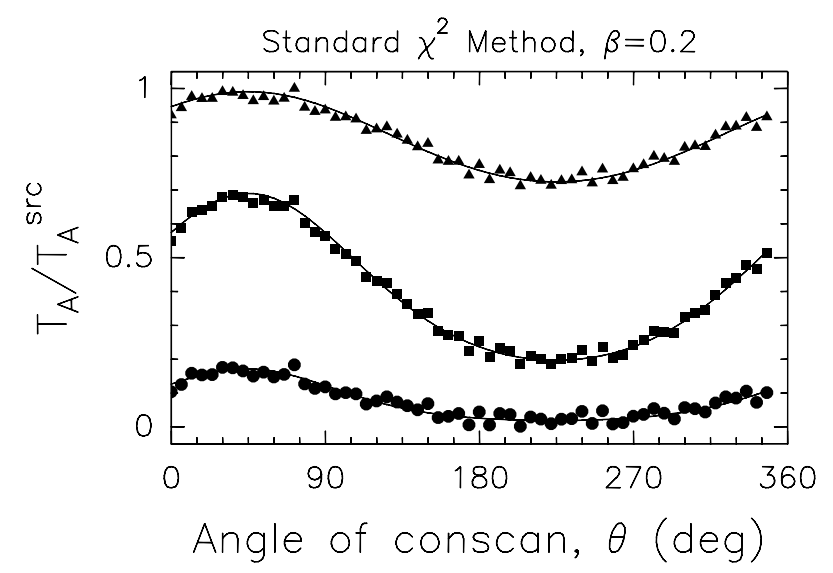

Fig. 3. Example of fit to the antenna signal, during 6-second scans, utilising the $\chi^{2}$ method (solid lines) for three different target positions: $X_{\mathrm{T}}=Y_{\mathrm{T}}=0.1$ (filled triangles), $X_{\mathrm{T}}=Y_{\mathrm{T}}=0.4$ (filled squares), and $X_{\mathrm{T}}=Y_{\mathrm{T}}=0.7$ (filled circles). The radius of the conical scan has been held constant, $\beta=0.2$. The data shown are for $\Delta t=0.1 \mathrm{~s}$, and $\Delta \theta=6^{\circ}$. A $1 \sigma$ noise is superimposed on the signal. The $0.25 \mathrm{Jy}$ calibration source is assumed to have $T_{\mathrm{A}}^{\mathrm{src}}=2.5 \mathrm{~K} \simeq 37 \sigma$

\section{Results}

We now describe the results of simulations where artificially generated antenna signals are analysed with the different methods illustrated above (Sect. 3). In doing this we assume first a Gaussian beam, as we did in Sect. 2, and will then investigate the case of a non-Gaussian antenna beam.

\subsection{Simulations with a Gaussian beam}

In this section we shall assume that the antenna beam is Gaussian and we shall almost always consider relatively small target displacements $\left(X_{\mathrm{T}}, Y_{\mathrm{T}} \lesssim 0.5\right)$ because when the pointing errors are much larger other pointing techniques (e.g., cross-scan) are more suitable than conical scanning.

Before actually showing the results of the individual techniques, we shall estimate the root-mean-square (rms) noise, $\Delta T_{\text {rms }}$, used in the aforementioned simulations. Assuming a $0.25 \mathrm{Jy}$ pointing source, an antenna gain of $10 \mathrm{~K} / \mathrm{Jy}$ (Arecibo approximate gain in $L$-band), and an integration time as short as $\Delta t=0.1 \mathrm{~s}$, one gets $\Delta T_{\text {rms }} \simeq 67 \mathrm{mK}$, if $\Delta \nu=2 \mathrm{MHz}$ and $T_{\mathrm{sys}}=25 \mathrm{~K}$. For a $T_{\mathrm{A}}^{\mathrm{src}}=2.5 \mathrm{~K}$ source this implies a $\mathrm{SNR} \simeq 37$. However, in the simulations discussed below we shall use lower SNRs to show the performance of the various retrieving methods in less favourable conditions.
Comparison of the true and calculated pointing errors is evaluated by computing the residual pointing error (in units of full beamwidth) defined as:

$e_{\mathrm{p}}=\sqrt{\left(X_{\mathrm{T}}-X_{\mathrm{T}}^{\mathrm{fit}}\right)^{2}+\left(Y_{\mathrm{T}}-Y_{\mathrm{T}}^{\mathrm{fit}}\right)^{2}}$.

The simulated noise is obtained using a random number generator and then added to the antenna signal. The retrieving procedure is carried out with this noisy signal and then repeated several times, adding a different random noise at each run. Finally, a mean value of the residual pointing error, $\left\langle e_{\mathrm{p}}\right\rangle$, is obtained.

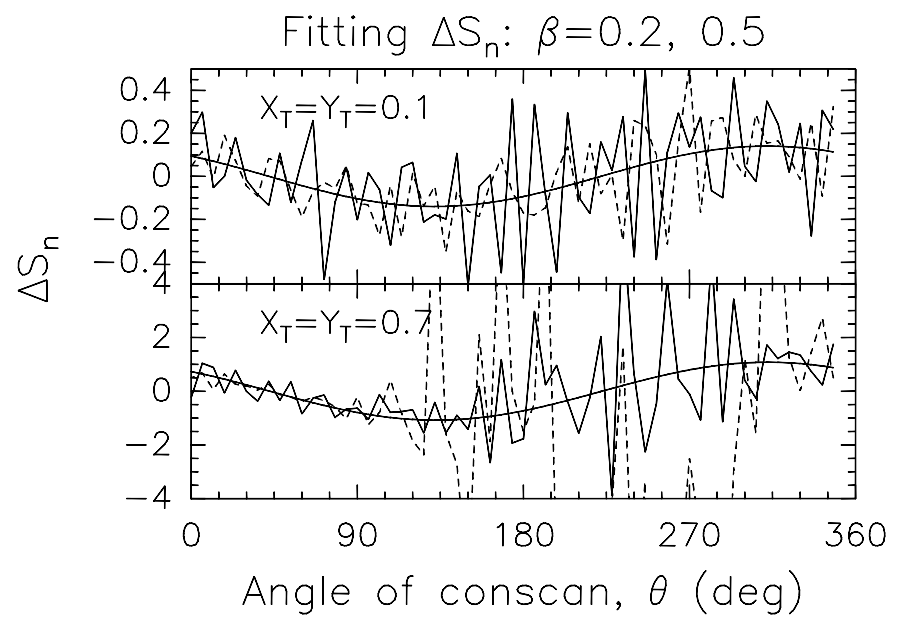

Fig. 4. Top: Example of fit to the normalized signal difference, $\Delta S_{\mathrm{n}} . \beta=0.2$ (solid line) and $\beta=0.5$ (dashed line), $\Delta t=1 \mathrm{~s}$, and $\Delta \theta=6^{\circ}$. The true values for the pointing errors in this simulation are $X_{\mathrm{T}}=Y_{\mathrm{T}}=0.1$, whereas the estimates from the fit (smooth solid line) are $X_{\mathrm{T}}=0.105$ and $Y_{\mathrm{T}}=0.094$. Bottom: Same as before, but with true values $X_{\mathrm{T}}=Y_{\mathrm{T}}=0.7$, whereas the estimates from the fit are $X_{\mathrm{T}}=0.79$ and $Y_{\mathrm{T}}=0.73$

\subsubsection{The standard numerical fitting method}

In Fig. 3 we show an example of application of the standard (non-linearized) fitting method to the antenna signal of the source, $T_{\mathrm{A}}(r)$, as described in the first part of Sect. 3.1. When compared to Fig. 4 (see Sect. 4.1.2) it is evident that the data to be fit when the antenna signal, rather than the normalised signal difference, is used, are much less noisy. As one can see, in Fig. 3 the $1 \sigma$ noise added to the signal can barely be seen.

It is also important to note that if only the peak-topeak response were measured, the conical scan output could give rise to ambiguos results in terms of the target position, because the modulation amplitude is zero for both zero and very large pointing errors, and rises to a maximum in between. This is shown in Fig. 3, where the peak-to-peak amplitudes of the $X_{\mathrm{T}}=Y_{\mathrm{T}}=0.1$ and $X_{\mathrm{T}}=Y_{\mathrm{T}}=0.7$ response curves do not differ greatly. 
There is however no ambiguity when the off-source zero level is known (see Sect. 2), as in Fig. 3.

When Eqs. (7) or (9) are used then, as we have seen in Sect. 3.1, the resulting linearized standard method makes maximum use of the available signal. However, the appropriate integration time in the radiometer equation is only one half of the conical scan period. In fact, two independent parameters, $X_{\mathrm{T}}$ and $Y_{\mathrm{T}}$, are to be determined and it can be shown that the weighted mean value of the variance of the fit is directly proportional to the number of parameters to be estimated (Richter 1995). As a consequence, the noise contribution must be calculated as if we were observing with an "effective integration time" which is half the duration of a single continuous conical scan.

In principle, sources with flux density $F_{\nu}^{\text {src }} \lesssim 0.1 \mathrm{Jy}$ can be used as pointing calibrators; assuming a system equivalent flux density of $F_{\nu}^{\mathrm{sys}}=2.5 \mathrm{Jy}$ and a bandwidth of $100 \mathrm{MHz}$, then the rms flux density becomes $\Delta F_{\nu}=$ $0.25 \mathrm{mJy}$, with a $4 \mathrm{~s}$ effective integration time (see also below). This gives a 800:1 SNR on boresight. However, depending on the frequency of observation and the RFI situation, the bandwidth to be used could also be as small as $2 \mathrm{MHz}$. But, even in this case, we still get a SNR of about $100: 1$.

In practice, atmospheric anomalous refraction (Olmi 1995) and, to a lesser extent, gain instability are more likely than noise to limit the pointing accuracy; this is a strong argument for keeping the conical scan period short. At Arecibo, a triplet of conical scans, consisting of $\mathrm{OFF}-$ SOURCE, CALIBRATION, BORESIGHT, CON-SCAN, CORRECTION, CON-SCAN, CORRECTION, CONSCAN, BORESIGHT, OFF-SOURCE, CALIBRATION, should take less than a minute, if the conical scans take $8 \mathrm{~s}$ and the remaining steps $2 \mathrm{~s}$ each, with $2 \mathrm{~s}$ slew time between steps. However, given the uncertainty concerning the real magnitude of the anomalous refraction effects at Arecibo, extensive tests will be needed in the field.

This assumes that the feed arm and the Gregorian continually track the nominal source position, and that all offsets are done with turret and tertiary. Accuracy in the absence of gain instability, anomalous refraction and RFI should be consistently better than 0.01 FWHM for any standard calibrator. The sequence given above should reliably handle pointing errors up to one beamwidth, much larger than those expected to occur in practice.

The results obtained by averaging $e_{\mathrm{p}}$ over several runs, as explained at the beginning of this section, are shown in the top-left panel of Fig. $5(\mathrm{SNR} \simeq 25)$ where we plot $\log _{10}\left(<e_{\mathrm{p}}>\right)$ as a function of the true pointing errors $X_{\mathrm{T}}$ and $Y_{\mathrm{T}}$. In the simulation program, the integrals in Eqs. (10) have been substituted with the proper sums, and the other parameters are as those of Fig. 4.

We note that the region within the $\left\langle e_{\mathrm{p}}\right\rangle=0.1$ contour extends up to $\epsilon \simeq 0.35$, despite all caveats discussed in Sect. 3.1, and it would be about the same also for a lower SNR (e.g., SNR $\simeq 8$ ). However, a region with much

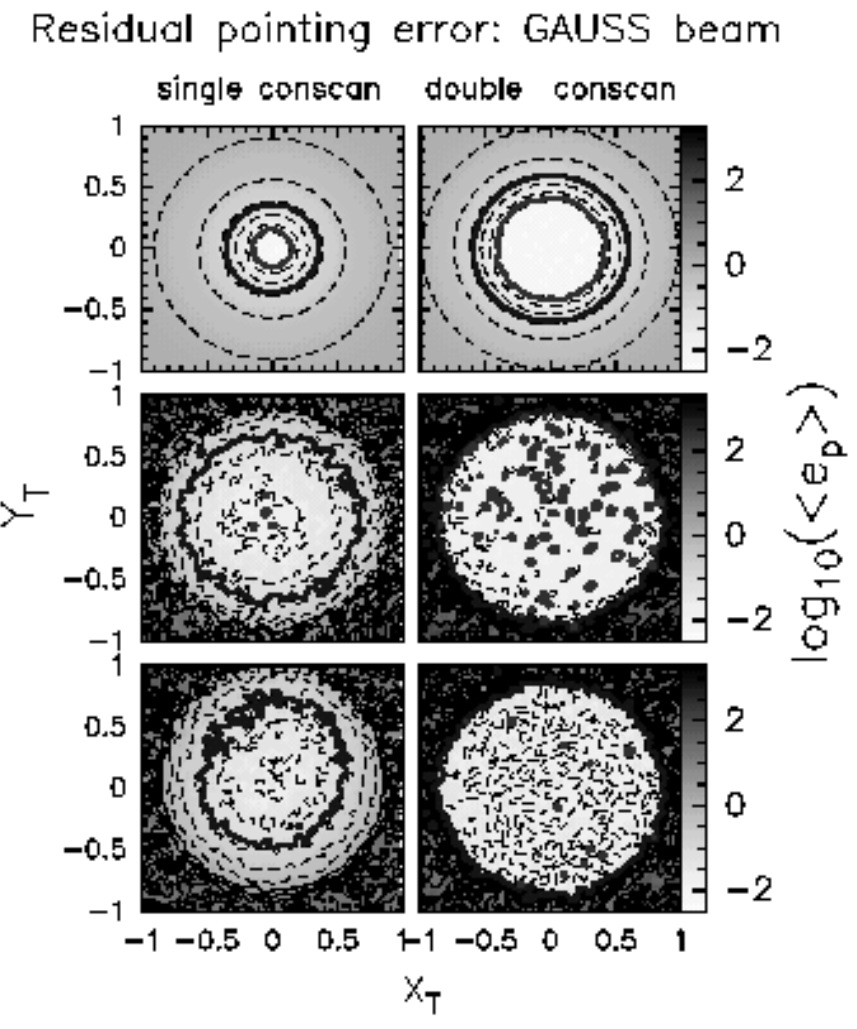

Fig. 5. Grey-scale map of $\log _{10}\left(<e_{\mathrm{p}}>\right)$ as a function of $X_{\mathrm{T}}$ and $Y_{\mathrm{T}}$, in the case of a SNR $\simeq 25$ and $\beta=0.2$. The figures on the left show the result of a single conscan, whereas those on the right were obtained after a second conscan was applied to the output of the first ones (see Sect. 4.1.4). Top: linearized standard method. Middle: fitting $\Delta S_{\mathrm{n}}$. Bottom: DP method. $\Delta t$ and $\Delta \theta$ are as in Fig. 4. Contour levels are from -2.5 to 3.3 by 0.4 , and negative values are plotted as dashed contours. The innermost and outermost thick contours in the top panels correspond to $e_{\mathrm{p}}=0.01$ and $e_{\mathrm{p}}=0.1$, respectively. In all other panels the longest and outermost thick contour corresponds to $e_{\mathrm{p}}=0.1$, whereas the smallest and randomly distributed thick contours correspond to $e_{\mathrm{p}}=0.01$. All figures have the same grey-scale contrast, so they can be directly compared

better estimates $\left(<e_{\mathrm{p}}>\leq 0.01\right.$, innermost thick contour in Fig. 5) is only found in the simulation with $\mathrm{SNR} \simeq 25$.

\subsubsection{Analytical methods: fitting the signal difference}

If the method of Sect. 3.2.1 is used, one can fit the measured values of $\Delta S_{\mathrm{n}}$ on one cycle by using the expression on the right hand side of Eq. (17). This procedure leads to estimates of $X_{\mathrm{T}}$ and $Y_{\mathrm{T}}$. In Fig. 4 we show an example of such a fit. The top panel shows the case of a small pointing error, $X_{\mathrm{T}}=Y_{\mathrm{T}}=0.1$, and with $\beta$ assuming two different values, 0.2 and 0.5 . In the bottom panel of Fig. 4 a similar example is shown, but for a larger pointing error, $X_{\mathrm{T}}=Y_{\mathrm{T}}=0.7$. In our test we have also added a $1 \sigma$ noise to the source signal, as described above. One can clearly see in this case the strong contribution of the noise (which 
depends on $\beta$ ), which does not prevent the fit from giving a good estimate of $X_{\mathrm{T}}$ and $Y_{\mathrm{T}}$, however. The residual pointing error $e_{\mathrm{p}}$ is $7.510^{-3}$ in the first case, and $9.810^{-2}$ in the second one.

In the middle-left panel of Fig. 5 we plot $\log _{10}\left(<e_{\mathrm{p}}>\right)$ as a function of the true pointing errors $X_{\mathrm{T}}$ and $Y_{\mathrm{T}}$, in the same conditions as those of Fig. 4 and fixing $\beta=0.2$. Using a lower $\operatorname{SNR}$ (e.g., $\simeq 8$ ) one finds that the parameters region within the $\left\langle e_{\mathrm{p}}\right\rangle=0.1$ contour would considerably shrink. Furthermore, beyond the $\left\langle e_{\mathrm{p}}\right\rangle=0.1$ limit the residual pointing error increases quickly and irregularly.

We can also estimate the total time needed for a complete pointing check, which is $N \times\left(\Delta t+\Delta t_{\text {slew }}\right)$, where $\Delta t_{\text {slew }}$ is the time needed to move from one position to the next, roughly estimated to be of order $1 \mathrm{~s}$. Therefore, in the case represented by Fig. 4 the complete test would last approximately 2-3 minutes.

\subsubsection{Analytical methods: Dot-product}

When the DP method is used, the normalised signal difference must be multiplied by a sine-wave of proper phase in order to retrieve the target position, as detailed in Sect. 3.2.2. In the simulation we have carried out, the oscillating terms present in Eq. (18) have been eliminated integrating both left hand and right hand sides between 0 and $\pi$. After performing such an integration the factor $c$ in Eq. (19) becomes equal to $\pi$. The geometry of conical scanning also implies that whereas $\theta=0-\pi$ will be the less noisy interval in the case of $X_{\mathrm{T}}, Y_{\mathrm{T}}>0$ (see Figs. 1 and 4 ), it will be the noisiest part in the opposite case, i.e. $X_{\mathrm{T}}, Y_{\mathrm{T}}<0$.

This method, as well as the $\Delta S_{\mathrm{n}}$-fit, needs a complete scan cycle to estimate the angle errors. Furthermore, when filtering is carried out through the integration described above, the noise components of $\Delta S_{\mathrm{n}}$ which are added in this process can give rise to large errors in the final estimate of $X_{\mathrm{T}}$ and $Y_{\mathrm{T}}$.

As in the previous two cases, the output of the DP method is shown in the bottom-left panel of Fig. 5. The parameters region within the $\left\langle e_{\mathrm{p}}\right\rangle=0.1$ contour shows a similar behaviour to the fitting method, although in the DP case this contour is smaller.

\subsubsection{Comparison of the methods}

A performance comparison of the various methods described above can be made using Fig. 5. The series of figures on the right column of Fig. 5 have been obtained by using the output from the single conscan runs (left column in Fig. 5) to apply a first pointing correction, and then running the conical scan again. The rightmost column in Fig. 5 therefore shows the final results as a function of the initial pointing errors $X_{\mathrm{T}}$ and $Y_{\mathrm{T}}$, after a pair of con- secutive conical scans and pointing corrections. We note that:

(i) Fig. 5 shows the almost perfectly circular contour plots of the linearised standard method. This is quite a distinct feature from the fitting and DP methods, especially at very large $\epsilon$ values where the distribution of $\left\langle e_{\mathrm{p}}\right\rangle$ in the $X_{\mathrm{T}}-Y_{\mathrm{T}}$ plane becomes highly chaotic.

(ii) in the high SNR case shown in Fig. 5 the area of the parameter region within $\left\langle e_{\mathrm{p}}\right\rangle=0.1$ is more extended if the fitting or DP methods are used. This fact makes them, rather than the standard method, more suitable for use at large $(\epsilon>0.5)$ pointing errors. However, it is important to note that in the case of the standard method, after a second conical scan the region within $\left\langle e_{\mathrm{p}}\right\rangle=0.01$ is more uniform and extended than in the fitting or DP methods.

(iii) we find that in a lower $\mathrm{SNR}$ case $(\mathrm{SNR} \simeq 8)$ the performance of the three methods decreases considerably, although to a much lesser extent in the case of the standard method, if just one conical scan is carried out.

The performance of the three methods as a function of the SNR is shown in Table 1, where the average value of $\left\langle e_{\mathrm{p}}\right\rangle$ is calculated over a region having a radius $\epsilon=0.5$, in the case of the single-conscan simulations.

Table 1. Average values of $\left\langle e_{\mathrm{p}}\right\rangle$ calculated over an area with $\epsilon \leq 0.5$, for different SNRs and for the three methods discussed in the text

\begin{tabular}{lcccc}
\hline Method & $\mathrm{SNR}=2$ & $\mathrm{SNR}=4$ & $\mathrm{SNR}=8$ & $\mathrm{SNR}=16$ \\
\hline Linearized standard & 0.12 & 0.09 & 0.08 & 0.07 \\
Fitting $\Delta S_{\mathrm{n}}$ & 16.6 & 0.85 & 0.17 & 0.05 \\
Dot product & 10.0 & 0.91 & 0.22 & 0.07 \\
\hline
\end{tabular}

Table 2. Average standard deviations, $\sigma_{<e_{\mathrm{p}}>}$, calculated in the area within the $e_{\mathrm{p}}=0.1$ contour of Fig. 5

\begin{tabular}{lcc}
\hline Method & Single conscan & Double conscan \\
\hline Linearized standard & $8.510^{-4}$ & $7.810^{-4}$ \\
Fitting $\Delta S_{\mathrm{n}}$ & $4.210^{-3}$ & $3.010^{-3}$ \\
Dot product & $5.010^{-3}$ & $5.010^{-3}$ \\
\hline
\end{tabular}

Regarding the accuracy of the residual pointing errors estimated above, Table 2 shows the average, $\sigma_{\left.<e_{\mathrm{p}}\right\rangle}$, of the standard deviations calculated for each individual point within the $e_{\mathrm{p}}=0.1$ contour of Fig. 5 . The results indicate that the linearized standard method has (for $\epsilon<0.5$ ) both lower residual pointing errors and smaller uncertainty compared to the other two methods. There is also a 


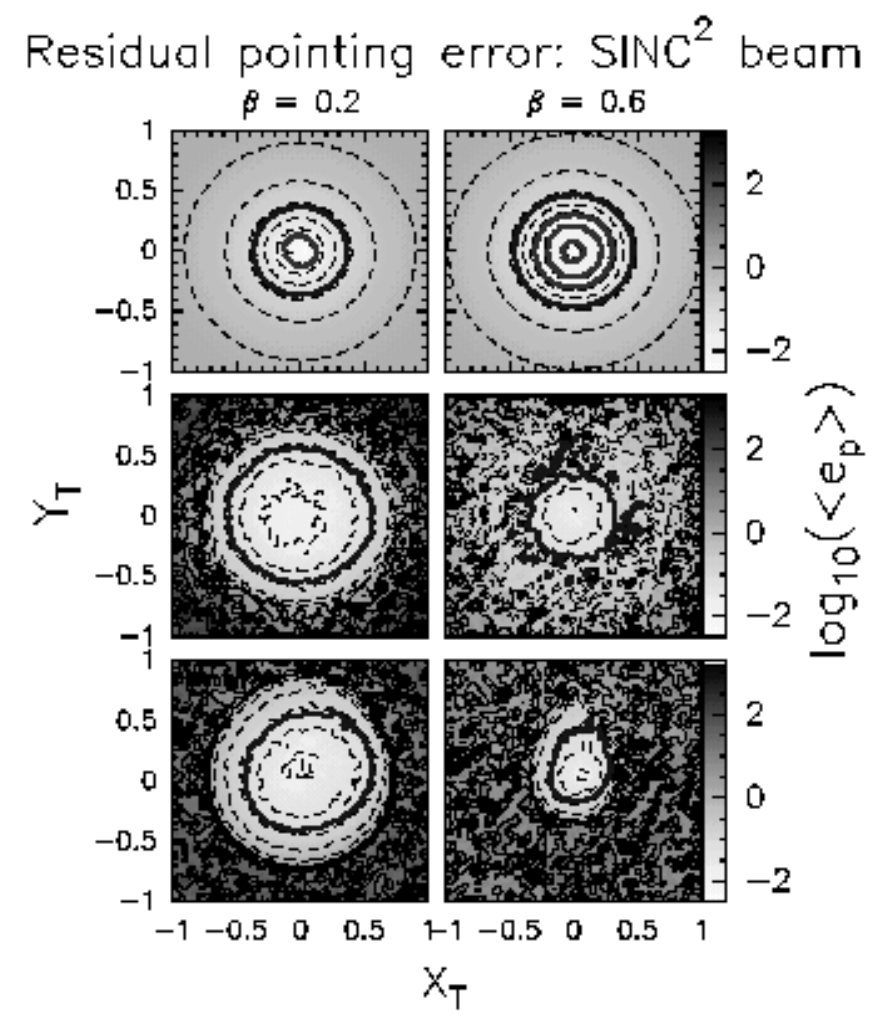

Fig. 6. Same as Fig. 5, for the data being generated using the $\sin c^{2}$ antenna beam described in the text and applying a single conscan only. The column on the left shows the results obtained with $\beta=0.2$, whereas the panels on the right-hand column correspond to $\beta=0.6$. The two different values of $\beta$ show the increasingly deteriorating effects of the sidelobes on the residual pointing error. Top: standard method. Middle: fitting $\Delta S_{\mathrm{n}}$. Bottom: DP method. The three innermost thick contours in the right-top panel all correspond to $e_{\mathrm{p}}=0.01$ (see also Fig. 7)

general decrease in the uncertainty after a second conscan has been carried out.

We conclude that in high SNR situations all methods can be used. However, one should expect better performance with the standard method at small $(\epsilon<0.5)$ initial pointing errors, and with the fitting and DP methods at large $(\epsilon \gtrsim 0.5)$ pointing errors. This is a consequence of the approximations used in Sect. 3.1, which make the linearized standard method less accurate when the initial pointing offset is large. Given the short time required at Arecibo to carry out a full conical scan, this is not however a strong limitation as a second conical scan can rapidly improve the pointing error estimation. With other less sensitive instruments the alternative method by Eldred (1994) may prove to be effective. On the other hand, as we mentioned in Sect. 4.1, the conical scan method should be used only when the pointing error is relatively small.

\subsection{Simulation with non-Gaussian beams}

A different situation arises when the simulation is carried out by generating the antenna signal assuming a nonGaussian beam. In this section we shall use the following shape for the beam of the antenna, which still has a circular cross-section in a plane perpendicular to the beam axis:

$T_{\mathrm{A}}(r)=T_{\mathrm{A}}^{\mathrm{src}}\left[\frac{\sin (0.886 \pi r)}{(0.886 \pi r)}\right]^{2}$.

Data generated using Eq. (21), with the usual addition of noise, can then be analysed with the methods discussed in Sect. 4.1.

Figure 6 shows the results of the application of the DP, linearized standard and $\Delta S_{\mathrm{n}}$-fitting methods to the data generated assuming the $\operatorname{sinc}^{2}$ cross-section defined above and applying a single conscan only. The simulation has been carried out for two different values of $\beta, 0.2$ and 0.6 . The purpose was to show the increasingly deteriorating effects of the sidelobes and the larger radius of the conical scan on the residual pointing error, $e_{\mathrm{p}}$.

A comparison with the results of Sect. 4.1 shows that the calculated estimates of $X_{\mathrm{T}}$ and $Y_{\mathrm{T}}$ are much less reliable when a relatively large conscan radius and a Gaussian beam shape are used to fit data obtained with a beam whose (symmetric) profile is not exactly known and has also sidelobes (see the extent of the $\left\langle e_{\mathrm{p}}\right\rangle=0.1$ contour in Fig. 6). However, the performance of the three methods is only slightly sensitive to changes in the beam shape when the conscan radius is small (at least when a Gaussian and a $\operatorname{sinc}^{2}$ beam are considered), as shown by the comparison of Figs. 5 and 6. Another and more general solution when the beam profile is expected to deviate strongly from a Gaussian would be to measure the beam shape, and then use the appropriate function for the numerical least-squares, as shown in Fig. 2.

The most interesting feature in Fig. 6 is the appearance of "ripples" in the distribution of the residual pointing errors for the standard method, in the case with $\beta=0.6$, due to the presence of sidelobes in the antenna beam. This is better shown in Fig. 7 which plots $\log _{10}\left(<e_{\mathrm{p}}>\right)$, for the standard method only, in the innermost part of the $\left(X_{\mathrm{T}}, Y_{\mathrm{T}}\right)$ plane. We have found that higher sidelobes would increase the residual pointing error but only at relatively large target displacements $(\epsilon \gtrsim 0.4)$.

\subsection{Limitations of conical scan and comparison to other methods}

In the proposed conical scan method the feed is moved relative to the antenna optical axis, so a number of comarelated effects may be introduced, as compared with other pointing methods where the feed is kept aligned with the antenna structure. We briefly discuss how they may affect the accuracy of conical scan below, highlighting the 


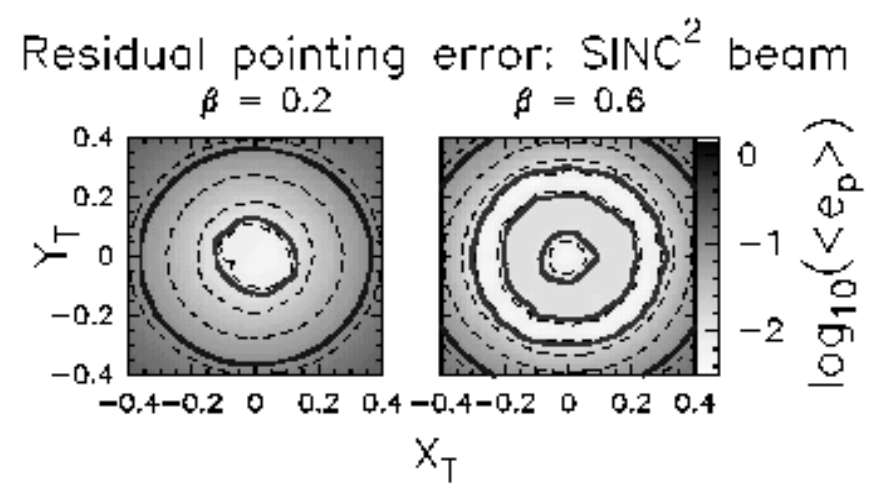

Fig. 7. Same as Fig. 6, linearized standard method only. This figure shows the innermost portion of Fig. 6 ; a different grey-scale is used (levels are from -2.5 to 0.2 by 0.4 ) to hightlight the presence of "ripples" in the distribution of the residual pointing errors in the case with $\beta=0.6$, due to the effect of sidelobes in the antenna beam. On the left, the innermost thick contour corresponds to $e_{\mathrm{p}}=0.01$, and the outermost is $e_{\mathrm{p}}=0.1$. On the right, the three innermost thick contours all correspond to $e_{\mathrm{p}}=0.01$, whereas the outermost is again $e_{\mathrm{p}}=0.1$

Arecibo case, and also compare the conical scan technique with the standard radio astronomy method of making cross-scans.

\subsubsection{Gain variation}

The lateral or axial displacement of the feed in a Cassegrain or Gregorian antenna system causes a boresight beam shift with a consequent loss of axial gain and beam degradation. At Arecibo the displacement of the feed horn off axis causes a modest decrease in performance, but much less than that experienced in some other shaped reflector systems (Kildal et al. 1993), limiting the field of view.

At $1.4 \mathrm{GHz}$ and above we expect a gain loss $\lesssim 12 \%$ at $1 \mathrm{FWHM}$ off axis (Kildal et al. 1993). This causes a negligible fluctuation in the signal level compared to the expected variation of the antenna temperature during a typical conical scan with $\beta=0.2$ and $\epsilon \simeq 0.5$ (see Figs. 2 and 3 ). This will be easily verified through a comparison with other methods during the commissioning phase of the conical scan method. We would expect this effect to be even more negligible in classical paraboloid systems with much more uniform subreflectors.

\subsubsection{Standing waves}

The formation of a standing-wave pattern caused by the interference of waves moving in parallel direction between the aperture plane of the feed horn and the apex of the main reflector may cause a baseline ripple in the observed spectra. However, at Arecibo we do not expect this to be a problem for several reasons.
First, the estimated peak to peak amplitude is expected to be less than $10 \mathrm{mK}$ (or about $1 \mathrm{mJy}$ ), i.e. much less than a "typical" $0.25 \mathrm{Jy}$ pointing source. Second, the conical scan method will be used in continuum mode, and thus the typical "wavelength" of the baseline ripple for the $132.6 \mathrm{~m}$ spacing between focus and reflector, about 1.1 MHz, will be usually significantly less than the measurement bandwidth. And finally, a value of beta can be selected to minimize the baseline ripple effect.

This may be a more important constraint for smaller antennas with wider ripple and lower SNR than Arecibo.

\subsubsection{Cross-polarization}

The cross-polarization at Arecibo comes from the offset nature of the dual reflector feed system, which introduces systematic cross-polarization for linear polarization, and from the platform blockage which introduces some random cross-polarization effects, at very low level. If the feed is displaced laterally from the focal point, and a dual circular polarization feed is used, then the right-hand beam points to a slightly different position in the sky than the left-hand beam.

However, the Gregorian design is expected to give cross-polarization levels in the aperture that are $-27 \mathrm{~dB}$ or lower for frequencies above $1 \mathrm{GHz}$, and therefore the onaxis beam squint is expected to be small. To measure any possible variation off-axis during the conical scan, both polarizations will be measured separately, to determine pointing differences. The beam squint will also be checked using alternative methods such as cross scans.

\subsubsection{Comparison to other pointing methods}

The proposed conical scan technique has great advantages over the standard radio astronomy pointing technique of making orthogonal scans on the nominal source position in the case of large ( $\gtrsim 40 \mathrm{~m}$ ) telescopes, as it does not require to move the massive (and slowly slewing) structure of the antenna, but only the feed support. Even when the mechanical nutation of a single feed is not included in the design, the large telescopes currently being planned or under construction, such as the NRAO "Green Bank Telescope" or the U.S./Mexican "Large Millimeter Telescope", have the potential to implement conical scan by displacing the subreflector or another mirror along the optical path.

When the level of the degradations discussed above can be appropriately controlled or removed, the conical scan method has also several other advantages, such as (see also Abichandani \& Ohlson 1981): (i) it uses all of the data all of the time, whereas the other schemes require larger overhead, making a more efficient use of the observing time; (ii) all pointing schemes have a degree of sensitivity to the asymmetry of the antenna beam profile, however the conical scan method has the least sensitivity as it averages around the entire scan; (iii) the standard 
cross-scan technique for pointing may cause unacceptable interruptions in data flow when the telescope is tracking a unique event or artificial satellite (Eldred 1994).

On the other hand, the standard pointing methods have the undoubted advantage that only the pointing parameters are involved in the retrieving technique, and that the computation requirement may be somewhat less than for some of the conical scan retrieving methods.

\section{Conclusions}

We have carried out a systematic analysis of a relatively unused method to point a radiotelescope, generally known as conical scan technique. It consists of moving the phase center of the feed along the circumference of a circle which is centered on the optical axis. The modulation of the signal returned from a point source that is off axis leads to an estimate of the pointing error.

This pointing technique has major advantages in the case of large radiotelescopes, such as the 305-m dish at Arecibo, where the feed turret and tertiary that will be used to move the feed in a circular path around the optical axis, are of very low mass compared with the 200-ton feed arm and 75-ton Gregorian enclosure. Also noticeable is the absence of tracking problems near zenith.

Three different methods have been discussed to retrieve the pointing corrections from a conical scan: (i) the standard fitting technique to the antenna signal; (ii) a fit to the normalised signal difference; and (iii) the dot-product method. The first one is a numerical technique that can be linearized and speeded up under the approximations of small pointing errors, in which case it becomes a very convenient analytical method. The other two are also analytical methods, and the dot-product technique, though less precise, can be implemented in hardware alone.

The results obtained with a Gaussian antenna beam indicate that during simulated observations of high SNR all methods are roughly equivalent, although at small $(\epsilon<0.5)$ pointing errors the linearised standard method is likely to give a better performance if the conical scan is repeated at least twice, in a consecutive way. Furthermore, the linearised standard method performs better at low SNRs, especially when conical scan is repeated more than once.

We have also carried out simulations with a sinc ${ }^{2}$ beam, to reproduce a situation where the (symmetric) antenna beam is not exactly known and some sidelobes may be present. In these cases the method fitting the signal difference and the dot-product method give a better performance at high SNRs and large pointing errors, whereas as usual the standard method gives better performance at small target displacements, although a general degradation is observed. However, contrary to the case of a Gaussian antenna beam, the standard method can now show some unwanted "ripples" in the distribution of the residual pointing error when the radius of the conical scan, $\beta$, is progressively increased. The method fitting the signal difference and the dot-product method are also deteriorated by the increased $\beta$ 's and the presence of the sidelobes.

\section{A. Fourier series and elliptical antenna-beams}

Following a standard approach we can expand $T_{\mathrm{A}}(\theta ; \beta, \epsilon)$ in a Fourier series of the type (we drop the explicit dependence on $\beta$ and $\epsilon$ on $T_{\mathrm{A}}$, for the sake of simplicity):

$T_{\mathrm{A}}(\theta)=\frac{I_{0}}{2}+\sum_{n=1}^{\infty} I_{n}^{\cos } \cos n \theta+\sum_{n=1}^{\infty} I_{n}^{\sin } \sin n \theta$

where the coefficients of the Fourier series are defined as:

$\left\{\begin{array}{l}I_{0}=\frac{1}{\pi} \int_{0}^{2 \pi} T_{\mathrm{A}}(\theta) \mathrm{d} \theta \\ I_{n}^{\cos }=\frac{1}{\pi} \int_{0}^{2 \pi} T_{\mathrm{A}}(\theta) \cos n \theta \mathrm{d} \theta \\ I_{n}^{\sin }=\frac{1}{\pi} \int_{0}^{2 \pi} T_{\mathrm{A}}(\theta) \sin n \theta \mathrm{d} \theta .\end{array}\right.$

If we now assume that $T_{\mathrm{A}}(\theta)$ can be approximated by the expansion to first order shown in Eq. (7), it can be easily shown that:

$I_{0}=2 T_{\mathrm{A}}^{\mathrm{src}} \exp \left[-k\left(\beta^{2}+\epsilon^{2}\right)\right]$.

Hence, $T_{\mathrm{A}}(\theta)$ can also be written as:

$T_{\mathrm{A}}(\theta)=\frac{I_{0}}{2}+I_{1}^{\cos } \cos \theta+I_{1}^{\sin } \sin \theta$

where all other coefficients of the Fourier series, $I_{n}^{\text {cos }}$ and $I_{n}^{\text {sin }}$, are 0 for $n \geq 2$, as a natural consequence of the special form of Eq. (7).

We now briefly discuss the case of an elliptical antennabeam, and what useful information can be obtained through the Fourier analysis. If the beam is non-circular, then $P_{\mathrm{n}}$ can no longer be represented by Eqs. (1) or (6). Instead, the normalized Gaussian antenna power pattern becomes:

$P_{\mathrm{n}}(x, y)=\exp \left(-k_{1} x^{2}\right) \exp \left(-k_{2} y^{2}\right)$

where both $x$ and $y$ are expressed in units of the full beamwidth at half maximum along the $X$ direction, $\mathrm{FWHM}_{x}$. We then define the ratio, $f$, of the full beamwidths at half maximum along the $X$ and $Y$ directions:

$f=\frac{\mathrm{FWHM}_{x}}{\mathrm{FWHM}_{y}}$

hence one obtains $k_{1}=k=4 \ln 2$ and $k_{2}=k_{1} f^{2}$. For comparison with Sect. 2 we then calculate the quantity $P_{\mathrm{n}}(\Delta x, \Delta y)$, where $\Delta x=\left(X_{\mathrm{T}}-X_{\mathrm{c}}\right)$ and $\Delta y=\left(Y_{\mathrm{T}}-Y_{\mathrm{c}}\right)$ :

$P_{\mathrm{n}}(\Delta x, \Delta y)=\exp \left[-k_{1}\left(\beta^{2}+\epsilon^{2}\right)\right] \exp \left[k_{1}\left(1-f^{2}\right) \times\right.$

$\left.\left(\beta^{2} \sin ^{2} \theta+Y_{\mathrm{T}}^{2}\right)+2 k_{1} \beta\left(X_{\mathrm{T}} \cos \theta+f^{2} Y_{\mathrm{T}} \sin \theta\right)\right]$ 
where we have used Eqs. (2) and (8). From Eq. (A7) we can then expand $T_{\mathrm{A}}(\theta)$ to first order, analogously to what we did in Eq. (7):

$$
\begin{array}{r}
T_{\mathrm{A}}(\theta) \simeq T_{\mathrm{A}}^{\mathrm{src}} \exp \left[-k_{1}\left(\beta^{2}+\epsilon^{2}\right)\right]\left[1+k_{1}\left(1-f^{2}\right) \times\right. \\
\left.\quad\left(\beta^{2} \sin ^{2} \theta+Y_{\mathrm{T}}^{2}\right)+2 \beta k_{1}\left(X_{\mathrm{T}} \cos \theta+f^{2} Y_{\mathrm{T}} \sin \theta\right)\right] .
\end{array}
$$

If $f=1$ (circular beam) we see that Eqs. (A7) and (A8) reduce to Eqs. (6) and (7), respectively.

We can now calculate, from Eqs. (A2), the first five coefficients in the Fourier series:

$$
\left\{\begin{array}{l}
I_{0}=\frac{\pi c_{1}}{k_{1} \beta}\left[1+\frac{k_{1}\left(1-f^{2}\right) \beta^{2}}{2}+k_{1} Y_{\mathrm{T}}^{2}\left(1-f^{2}\right)\right] \\
I_{1}^{\cos }=\pi c_{1} X_{\mathrm{T}} \\
I_{1}^{\sin }=\pi c_{1} f^{2} Y_{\mathrm{T}} \\
I_{2}^{\cos }=-\frac{\pi c_{1} \beta\left(1-f^{2}\right)}{4} \\
I_{2}^{\sin }=0
\end{array}\right.
$$

where we have defined

$c_{1}=\frac{2 k_{1} \beta T_{\mathrm{A}}^{\mathrm{src}} \exp \left[-k_{1}\left(\beta^{2}+\epsilon^{2}\right)\right]}{\pi}$.

If $f=1$ Eqs. (A9) reduce to Eqs. (11) and (A3). We see that the coefficient $I_{2}^{\cos }$ is a measure of the ellipticity in the antenna-beam, as one can calculate $f$ from $I_{2}^{\cos }$ :

$f=\sqrt{1+\frac{4}{\pi c_{1} \beta} I_{2}^{\cos }}$

We note that $c_{1}$ contains the unknown, $\epsilon$; this potential problem has already been discussed in Sect. 3.1.

\section{B. Principal symbols}

$$
\begin{aligned}
& e_{\mathrm{p}} \quad \text { error difference between the true } \\
& \text { Eq. (20) }
\end{aligned}
$$
maximum along the $X$ and $Y$ directions
$P_{\mathrm{n}} \quad$ normalized antenna power pattern Eq. (1)

angular distance between the center of the antenna beam and the target position Eq. (3)

$R_{\mathrm{T}} \quad$ ratio of measured boresight antenna temperature to expected source antenna temperature

Eq. (14)

$\Delta S_{\mathrm{n}} \quad$ normalized signal difference between two successive positions of the antenna beam on the circular path

Eq. (17)

$T_{\mathrm{A}}(r) \quad$ measured antenna temperature of the pointing source

Eq. (4)

$T_{\mathrm{A}}^{\mathrm{src}} \quad$ expected antenna temperature of the pointing source at center of the antenna beam Eq. (5)

$W_{\text {on }}(r), W_{\text {off }}$ measured power ON-source and OFF-source,

$W_{\text {src }} \quad$ respectively; $W_{\text {src }}=W_{\text {on }}(r=0)$ Eq. (1)

$\left(X_{\mathrm{c}}, Y_{\mathrm{c}}\right) \quad$ cartesian coordinates of the beam center (cross-elevation and elevation)

Eq. (2)

$\left(X_{\mathrm{T}}, Y_{\mathrm{T}}\right) \quad$ cartesian coordinates of the target (cross-elevation and elevation) Eq. (8)

radius of the circular path described by the antenna beam center Eq. (2)

angular distance between the optical axis and the target

Eq. (8)

$\theta \quad$ angle defining the position of the center of the antenna beam with respect to the $X$ axis Eq. (2)

$\theta_{\epsilon} \quad$ angle defining the unknown position of the target with respect to the $X$ axis Eq. (8) instrumental phase offset used in the dot-product method Eq. (18) 
Acknowledgements. We are grateful to C.J. Salter and P.F. Goldsmith for critically reading the manuscript and useful advice regarding some aspects of conical scanning, and to the referee, M. Kesteven, for insightful comments.

\section{References}

Abichandani K.P., 1983, TDA Progress Report 42-73, JanuaryMarch 1983

Abichandani K.P., Ohlson J.E., 1981, TDA Progress Report 42-64, May and June 1981

Eldred D.B., 1994, TDA Progress Report 42-116, February 15 1994

Kildal P.-S., Johansson M. Hagfors T., Giovanelli R., 1993, IEEE Trans. Ant. Prop. 41, 1019

Kildal P.-S., Baker L., Hagfors T., 1994, Proceedings of the IEEE 82,714
Ohlson J.E., Abichandani J.E., 1982, TDA Progress Report 42-70, May and June 1982

Olmi L., November 1995, Internal Report, Arecibo Observatory

Olmi L., Baan W., 1997, Proceedings of the first NAIC Workshop on the "Molecular Spectroscopy in the 1 to $10 \mathrm{GHz}$ Range with the Upgraded Arecibo Telescope" held in Arecibo, PR (U.S.A.), 19 and 20 October, 1995, Astrophysical Letters \& Communications (in press)

Richter P.H., August, 1995, TDA Progress Report 42-122, April-June 1995

Skolnik M.I., 1970, Radar Handbook. McGraw-Hill, New York Sherman S.M., 1984, Monopulse Principles and Techniques. Artech House

Wark R.M., Wright A.E., 1990, Proc. ASA 8, 375 